\title{
SPLIT DOMINATION VERTEX AND EDGE CRITICAL GRAPHS
}

\author{
GIRISH V. R. ${ }^{1}$, P. Usha ${ }^{2}$ \\ ${ }^{1}$ Department of Science and Humanities, PES University(EC campus), \\ Electronic City, Bengaluru, Karnataka, India, \\ email: giridsi63@gmail.com \\ ${ }^{2}$ Department of Mathematics, Siddaganga Institute of Technology, \\ B.H. Road, Tumakuru, Karnataka, India, \\ email: ushapmurthy@yahoo.com
}

\begin{abstract}
A dominating set $D$ of a graph $G=(V, E)$ is a split dominating set if the induced graph $\langle V-D\rangle$ is disconnected. The split domination number $\gamma_{s}(G)$ is the minimum cardinality of a split domination set. A graph $G$ is called vertex split domination critical if $\gamma_{s}(G-v)<\gamma_{s}(G)$ for every vertex $v \in G$. A graph $G$ is called edge split domination critical if $\gamma_{s}(G+e)<\gamma_{s}(G)$ for every edge $e$ in $\bar{G}$. In this chapter, whether for some standard graphs are split domination vertex critical or not are investigated and then characterized $2-\gamma_{n s}$-critical and $3-\gamma_{n s}$-critical graphs with respect to the diameter of a graph $G$ with vertex removal. Further, it is shown that there is no existence of $\gamma_{s}$-critical graph for edge addition.
\end{abstract}

\section{INTRODUCTION}

The graphs considered in this paper are finite, connected, undirected and without loops or multiple edges. Terminology not defined here will conform to that in [4]. Let $P_{n}, C_{n}, K_{1, n}, K_{n}, K_{m, n}, W_{n}, D_{n}^{(m)}$ denote the path, cycle, star, complete graph, bipartite graph, wheel graph, and dutch wind mill graph. An end vertex of a graph $G$ is a vertex of degree 1 and an support vertex of a graph $G$ is a vertex adjacent to an end vertex.

The neighborhood of a vertex $v$ in $G$ is denoted by $N(v)$ and is given by $N(v)=\{u \in V(G) / u$ is adjacent to $v$ in $G\}$. The diameter of the graph $G$ is a measure of the length of the longest minimal path and is denoted by $\operatorname{diam}(G)$.

The graph with $n+1$ vertices labeled $u_{1}, u_{2}, u_{3}, u_{4}, \ldots, u_{n+1}$ and edges $u_{1} u_{2}$, $u_{2} u_{3}, u_{3} u_{4}, \ldots, u_{n} u_{n+1}$ is called a path of length $n$, denoted by $P_{n+1}$. We call $u_{1}$ and $u_{n+1}$ the end-vertices of the path. The cycle of length of $n, C_{n}$, is the graph with $n$ vertices $u_{0}, u_{1}, u_{2}, u_{3}, \ldots, u_{n-1}$ and the edges $u_{0} u_{1}, u_{1} u_{2}, u_{2} u_{3}, \ldots, u_{n-1} u_{0}$.

2020 Mathematics Subject Classification: 7500.

Received: 15-09-2018, accepted: 11-09-2019. 
A simple graph of order $n$ with all possible edges is called a complete graph of order $n$, denoted by $K_{n}$.

A graph $G=(V, E)$ is said to be $r$-partite (where $r$ is an positive integer) if its $V(G)$ can be partitioned as $V=V_{1} \cup V_{2} \cup, \ldots, \cup V_{r}$ such that if $u v$ is an edge of $G$ then $u$ is in some $V_{i}$ and $v$ is in some other $V_{j}$; that is, everyone of the induced subgraphs $\left\langle V_{i}\right\rangle$ is an empty graph. The graph obtained from $G$ by subdividing each edge of $G$ exactly once is called the subdivision graph [1] of $G$ and it denoted by $S(G)$.

A set of vertices $S$ is said to dominate the graph $G$ if for each $v \notin S$, there is a vertex $u \in S$ with $v$ adjacent to $u$. The minimum cardinality of any dominating set is called the domination number of $G$ and is denoted by $\gamma(G)$. For complete review on domination refer [5]. The concept of split domination has been studied by V.R. Kulli and B. Janikiram [6]. A dominating set $D$ of a graph $G=(V, E)$ is a split dominating set if the induced graph $\langle V-D\rangle$ is disconnected. The split domination number $\gamma_{s}(G)$ is the minimum cardinality of a split domination set.

A graph $G$ is called vertex split critical if $\gamma_{s}(G-v)<\gamma_{s}(G)$ for every vertex $v$ in $G$. Thus, $\mathrm{G}$ is $k-\gamma_{s}$-critical if $\gamma_{s}(G)=k$, for each vertex $v \in V(G), \gamma_{s}(G-v)<k$. A graph $G$ is called edge split critical if $\gamma_{s}(G+e)<\gamma_{s}(G)$ for every edge $e$ in $\bar{G}$. Thus, $G$ is k- $\gamma_{s}$-critical if $\gamma_{s}(G)=k$ and for each edge $e \in \bar{G}, \gamma_{s}(G+e)<k$.

Let us consider the two terrorist groups say A and B which are interconnected with each other with group members as the vertices and the edges as the communication between them. Among these two groups, their are minimum number of people who had the communication with all the members of the two groups called domination members, among them few may have communication between two groups. Since the two terrorist groups are connected their terrorist activity may increase. Suppose the military people wants to make the terrorists activity to be inactive, it is better to destroy the domination members and also the groups members in such a way that the two groups gets separated so that there is no communication between the groups and also between the members of the groups. This is the motivation for studying split domination.

The domination vertex and edge critical graphs studied extensively in $[7,2$, $8,3,9]$. Since the dominating members are more strong, instead of dominating members, we can destroy the others members along with the dominating members in such a way that, no communication between the two groups. The numbers of people to be destroyed will be less than or equal to the domination members. This motivated us to study split domination vertex and edge critical graphs. First we discuss whether some particular classes of graphs are $\gamma_{s}$-vertex critical or not, $2-\gamma_{s}$-vertex critical and $3-\gamma_{s}$-vertex critical graphs are characterized with respect to diameter of the graph $G$ for vertex removal and then we had shown that there is no $\gamma_{s}$-critical graph for edge addition. 


\section{SPLit DOMINATION VERTEX CRITICAL GRAPHS}

In this entire section, the graph $G \neq K_{n}$, considered should be a simple graph and $G-\{v\}, v \in V(G)$ having $n$ components, either contains a non-complete component or at least two non-trivial components.

Theorem 2.1. (Kulli and Janakiram [6]) For any cycle $C_{n}, \gamma_{s}\left(C_{n}\right)=\left\lceil\frac{n}{3}\right\rceil, n \geq 4$.

Theorem 2.2. (Chelvam and Chellathurai $[10]) \gamma_{s}\left(P_{n}\right)=\left\lceil\frac{n}{3}\right\rceil, n>2$, where $P_{n}$ is a path of length $n-1$.

Theorem 2.3. (Kulli and Janakiram [6]) For any connected graph $G$ with an end-vertex, $\gamma_{s}(G)=\gamma(G)$. Furthermore, there exists a $\gamma_{s}$-set of $G$ containing all vertices adjacent to end-vertices.

Theorem 2.4. For any connected graph $G$,

$$
\gamma_{s}(G)-1 \leq \gamma_{s}(G-v) \leq \gamma_{s}(G)+\operatorname{deg}(v)-1, v \in V(G)
$$

Proof. Let $G$ be a connected graph and $v \in V(G)$. Since the domination number will increase by more than one and decreases by at most one when a vertex is removed from $G$, thus $\gamma_{s}(G)-1 \leq \gamma_{s}(G-v)$. For the upper bound, let $H$ be a $\gamma_{s}$-set of $G$.

Case 1. Let $v \notin H$, then $\gamma_{s}(G-v) \leq \gamma_{s}(G)$.

Case 2. Let $v \in H$, and let $B=\left\{v_{i} / v_{i} \in N(v), v_{i} \notin N(H-v)\right\}$.

(i) If $|B|=\phi$, then $\gamma_{s}(G-v)=|H|-|v|$. Hence $\gamma_{s}(G-v)<\gamma_{s}(G)$.

(ii) If $|B| \neq \phi$, then $\gamma_{s}(G-v) \leq|H|+|B|-|\{v\}|=\gamma_{s}(G)+|B|-1$.

Since $|B| \leq \operatorname{deg}(v), \gamma_{s}(G-v) \leq \gamma_{s}(G)+\operatorname{deg}(v)-1$.

Hence the proof.

Theorem 2.5. The graph $G=C_{n}, n>3$ is $\gamma_{s}$-vertex critical for $n=3 p+1, p \geq 1$ and is not $\gamma_{s}$-vertex critical for $n \neq 3 p+1$.

Proof. By Theorem 2.1, $\gamma_{s}\left(C_{n}\right)=\left\lceil\frac{n}{3}\right\rceil$ and in $G-\{v\}, v \in V(G)$ will be a path with $n-1$ vertices and by Theorem 2.2 , for $n-1$ vertices, $\gamma_{s}(G-v)=\gamma_{s}\left(P_{n-1}\right)=$ $\left\lceil\frac{n-1}{3}\right\rceil$.

Case 1. When $n=3 p+1, p \geq 1$.

Then, $\gamma_{s}(G-v)=\gamma_{s}\left(P_{n-1}\right)=\left\lceil\frac{3 p+1-1}{3}\right\rceil=\lceil p\rceil$. Since $\lceil p\rceil<\left\lceil p+\frac{1}{3}\right\rceil$ for any $p \geq 1$. Thus $\gamma_{s}(G-v)<\gamma_{s}(G)$. Hence $G=C_{n}$ is $\gamma_{s}$-vertex critical for $n=3 p+1, p \geq 1$. Case 2. When $n \neq 3 p+1, p \geq 1$.

Since $\left\lceil\frac{n}{3}\right\rceil=\left\lceil\frac{n-1}{3}\right\rceil$ for $n=3 p+2$ or $n=3 p+3, p \geq 1$. Thus $\gamma_{s}(G-v)=\gamma_{s}(G)$. Hence $G=C_{n}$ is not $\gamma_{s}$-vertex critical for $n \neq 3 p+1, p \geq 1$.

Theorem 2.6. The path $P_{n}, n \geq 5$ is not $\gamma_{s}$-vertex critical.

Proof. Case 1. If $v_{i}$ is not an end-vertex, then $P_{n}-\left\{v_{i}\right\}$ is disconnected into two components $G_{1}$ and $G_{2}$ with $\left|V\left(G_{1}\right)\right|=n_{1}$ and $\left|V\left(G_{2}\right)\right|=n_{2}$ such that $n_{1}+n_{2}+1=$ $n$. For a disconnected graph, $\gamma_{s}(G)=\gamma(G)$, thus $\gamma_{s}\left(P_{n}-v_{i}\right)=\gamma\left(G_{1}\right)+\gamma\left(G_{2}\right)$. Since $G_{1}$ and $G_{2}$ are paths.

Subcase 3.1. When $\left(n_{1} \neq 3 r\right.$ and $\left.n_{2} \neq 3 r\right)$ or $\left(n_{1} \neq 3 r\right.$ and $\left.n_{2}=3 r\right)$ or $\left(n_{1}=3 r\right.$ and $n_{2} \neq 3 r, r \geq 1$ ), 
Thus $\gamma_{s}\left(P_{n}-v_{i}\right) \geq \gamma_{s}\left(P_{n}\right)$.

$$
\begin{aligned}
\gamma_{s}\left(P_{n}-v_{i}\right) & =\left\lceil\frac{n_{1}}{3}\right\rceil+\left\lceil\frac{n_{2}}{3}\right\rceil \\
& \geq\left\lceil\frac{n_{1}+n_{2}}{3}\right\rceil \\
& \geq\left\lceil\frac{n_{1}+n-1-n_{1}}{3}\right\rceil \\
& \geq\left\lceil\frac{n-1}{3}\right\rceil=\left\lceil\frac{n}{3}\right\rceil
\end{aligned}
$$

Subcase 3.2. When $n_{1}=3 r$ and $n_{2}=3 r, r \geq 1$,

$$
\begin{aligned}
\gamma_{s}\left(P_{n}-v_{i}\right) & =\left\lceil\frac{n_{1}}{3}\right\rceil+\left\lceil\frac{n_{2}}{3}\right\rceil \\
& =\frac{n_{1}+n_{2}}{3} \\
& =\frac{n_{1}+n-1-n_{1}}{3} \\
& =\frac{n-1}{3}
\end{aligned}
$$

Since $\frac{n-1}{3}<\left\lceil\frac{n}{3}\right\rceil$. Thus $\gamma_{s}\left(P_{n}-v_{i}\right)<\gamma_{s}\left(P_{n}\right)$. $P_{n}$ in not $\gamma_{s}$-critical because $\gamma_{s}\left(P_{n}-v_{i}\right)<\gamma_{s}\left(P_{n}\right)$ only if $v_{i}$ is not an end-vertex and $n_{1}=3 r, n_{2}=3 r$ not for any vertex $v_{i} \in V\left(P_{n}\right)$

Case 2. Suppose $v_{i}$ is an end-vertex of $P_{n}$ and $n=3 p+1, p \geq 2$. By Theorem 2.2, $\gamma_{s}\left(P_{n}\right)=\left\lceil\frac{3 p+1}{3}\right\rceil=\left\lceil p+\frac{1}{3}\right\rceil$. Thus $\gamma_{s}\left(P_{n}-v_{i}\right)=\gamma_{s}\left(P_{n-1}\right)=\left\lceil\frac{n-1}{3}\right\rceil=\left\lceil\frac{3 p+1-1}{3}\right\rceil=$ $\lceil p\rceil$. Since $\lceil p\rceil<\left\lceil p+\frac{1}{3}\right\rceil=\left\lceil\frac{n}{3}\right\rceil$ for $p \geq 2$. Hence $\gamma_{s}\left(P_{n}-v_{i}\right)<\gamma_{s}\left(P_{n}\right)$. $P_{n}$ in not $\gamma_{s}$-critical because $\gamma_{s}\left(P_{n}-v_{i}\right)<\gamma_{s}\left(P_{n}\right)$ only if $v_{i}$ is end-vertex not for any vertex $v_{i} \in V\left(P_{n}\right)$

Case 3. Suppose $v_{i}$ is an end-vertex and $n \neq 3 p+1, p \geq 2$. By Theorem 2.2, $\gamma_{s}\left(P_{n}\right)=\left\lceil\frac{n}{3}\right\rceil$. Thus $\gamma_{s}\left(P_{n}-v_{i}\right)=\gamma_{s}\left(P_{n-1}\right)=\left\lceil\frac{n-1}{3}\right\rceil$. Since $\left\lceil\frac{n}{3}\right\rceil=\left\lceil\frac{n-1}{3}\right\rceil$ for $n=3 p+2$ or $n=3 p+3, p \geq 1$. Hence $\gamma_{s}\left(P_{n}-v_{i}\right)=\gamma_{s}\left(P_{n}\right)$.

From all the cases above, $P_{n}, n \geq 5$ is not $\gamma_{s}$-vertex critical.

Theorem 2.7. If a graph $G$ is $2-\gamma_{s}$-vertex critical, then $\operatorname{diam}(G)=2$.

Proof. Let $G$ be a connected $2-\gamma_{s}$-vertex critical graph and suppose $G$ has diameter at least 3. Assume that $P=v_{1}, v_{2}, \ldots, v_{d}$ be the longest diametrical path with the distance equal to the $\operatorname{diam}(G)$. Let $D^{1}$ be a $\gamma_{s}$-set of $G-\{v\}, v \in$ diametrical path. Since $G$ is $2-\gamma_{s}$-vertex critical graph, then $\gamma_{s}(G-v)=1$ for any vertex $v \in V(G)$ and $\left|D^{1}\right|=1$. If $v_{k} \in D^{1} \cap P$, then there exists at least one vertex say $v_{j} \in$ diametrical path in $G-\{v\}$ which is not covered by $v_{k}$. Thus $v_{j}$ is not dominated by any vertex of $D^{1}$, which is a contradiction. If $\operatorname{diam}(G)$ is one, then $G$ is not $\gamma_{s}$-vertex critical.

Theorem 2.8. If a connected graph $G$ is $3-\gamma_{s}$-vertex critical, then $\operatorname{diam}(G) \leq 4$.

Proof. Let $G$ be a connected $3-\gamma_{s}$-vertex critical graph and suppose $G$ has diameter at least 5 . Assume that $P=v_{i}, i=1,2,3, \ldots, d$ be the longest diametrical path with its distance equal to the $\operatorname{diam}(G)$. Let $D_{1}$ be a $\gamma_{s}$-set of $G-\{v\}, v \in$ diametrical path. Since $G$ is $3-\gamma_{s}$-vertex critical, then $\gamma_{s}(G-v) \leq 2$ for any vertex $v \in V(G)$. The set $D_{1}$ contains at most two vertices say $v_{1}$ and $v_{2}$.

Case 1: If both $v_{1}$ and $v_{2}$ are in $D_{1}$, then there exists at least one vertex say $v_{r} \in$ diametrical path in $G-\{v\}$ which is not adjacent to $v_{1}$ or $v_{2}$ and hence a contradiction.

Case 2: If either of $v_{1}$ or $v_{2}$ is in $D_{1}$, then there exists at least one vertex say $v_{k}$ in 
$G-\{v\}$ which is not covered by $v_{1}$ or $v_{2}$, which is a contradiction.

This completes the proof.

Theorem 2.9. No tree $T, n \geq 4$ is $\gamma_{s}$-vertex critical with respect to split domination.

Proof. Let $D$ be a $\gamma_{s}$-set of tree $T, A=\left\{v_{i} / v_{i}\right.$ is a support vertex of $\left.T\right\}$, then by Theorem 2.3, the set $D$ will not contain an end-vertices, $A \subseteq D$. Now consider the graph $T-\{v\}, v \in A$. The graph $T-\{v\}$ is disconnected into $k \geq 2$ components and let $C=\left\{v_{m} / v_{m} \in N(v), v_{m} \notin N(D-v)\right\}$.

Case 1. If $|C|=1$, then $\gamma_{s}(T-v)=|D|-|\{v\}|+|C|=|D|=\gamma_{s}(T)$.

Case 2. if $|C| \neq 1$, then $\gamma_{s}(T-v)=|D|-|\{v\}|+|C|>|D|=\gamma_{s}(T)$.

Hence the tree $T$ is not $\gamma_{s}$-vertex critical.

Proposition 2.10. For any Wheel graph $W_{n}, \gamma_{s}\left(W_{n}\right)=3$.

Theorem 2.11. The graph $G=W_{n}$ is $\gamma_{s}$-vertex critical for $n=5,6,7$ and not $\gamma_{s}$-vertex critical for $n \geq 8$.

Proof. Let $v_{j} \in V(G)$ such that $\operatorname{deg}\left(v_{j}\right)=n-1$ and let $D$ be a $\gamma_{s}$-set of $G=W_{n}$. Case 1. $n=5,6,7$.

Let $v \in V(G), v \neq v_{j}$, then $\gamma_{s}(G-v)=\left|\left\{v_{j}\right\} \cup\left\{v_{k}\right\}\right|=2, v_{k} \notin N(v)$ in $G$. By Proposition 2.10, $\gamma_{s}(G-v)<\gamma_{s}(G)$. If $v=v_{j}$, then $G-\left\{v_{j}\right\}$ will be a cycle $C_{4}$ or $C_{5}$ or $C_{6}$ and $\gamma_{s}\left(C_{4}\right.$ or $C_{5}$ or $\left.C_{6}\right)=2$. By Proposition $2.10, \gamma_{s}(G-v)<\gamma_{s}(G)$. Hence $G=W_{n}$ is $\gamma_{s}$-vertex critical for $n=5,6,7$.

Case 2. $n \geq 8$.

Let $v \in V(G), v \neq v_{j}$, then $\gamma_{s}(G-v)=\left|\left\{v_{j}\right\} \cup\left\{v_{k}\right\}\right|=2, v_{k} \notin N(v)$ in $G$. By Proposition 2.10, $\gamma_{s}(G-v)<\gamma_{s}(G)$. If $v=v_{j}$, then $G-\left\{v_{j}\right\}$ will be a cycle $C_{n}, n \geq 7$ and $\gamma_{s}\left(C_{n}\right) \geq 3$. By Proposition 2.10, $\gamma_{s}(G-v) \geq \gamma_{s}(G)$. Hence $G=W_{n}$ is not $\gamma_{s}$-vertex critical for $n \geq 8$. This completes the proof.

Definition 2.12. The Dutch wind mill graph $D_{n}^{(m)}$ is the graph obtained by taking $m$ copies of the cycle $C_{n}$ with a vertex in common.

Proposition 2.13. For any Dutch wind mill graph $D_{n}^{(m)}, m \geq 2, n \geq 4, \gamma_{s}\left(D_{n}^{(m)}\right)=$ $m\left\lceil\frac{n-3}{3}\right\rceil+1$.

Theorem 2.14. The Dutch wind mill graph $G=D_{n}^{(m)}, m \geq 2, n \geq 4$, is $\gamma_{s}$-vertex critical for $n=3 p+1, p \geq 1$ and not $\gamma_{s}$-vertex critical for $n \neq 3 p+1, p \geq 1$.

Proof. Let $v_{k} \in V\left(D_{n}^{(m)}\right)$ with $\operatorname{deg}\left(v_{k}\right)=2 m$.

Case 1. If $v=v_{k}$, then $G-\left\{v_{k}\right\}$ is disconnected into $m$ components, where each component is of $P_{n-1}$. By Theorem 2.2, $\gamma_{s}\left(G-v_{k}\right)=m\left\lceil\frac{n-1}{3}\right\rceil$.

Subcase 1.1. If $n=3 p+1, p \geq 1$, then $m\left\lceil\frac{n-1}{3}\right\rceil=m\left\lceil\frac{n-3}{3}\right\rceil, m \geq 2, n \geq 4$. Hence by Proposition 2.13, $\gamma_{s}\left(G-v_{k}\right)<\gamma_{s}(G)$.

Subcase 1.2. If $n \neq 3 p+1, p \geq 1$, then $m\left\lceil\frac{n-1}{3}\right\rceil>m\left\lceil\frac{n-3}{3}\right\rceil, m \geq 2, n \geq 4$. Hence by Proposition 2.13, $\gamma_{s}\left(G-v_{k}\right)>\gamma_{s}(G)$.

Case 2. If $v \neq v_{k}$, then $G-v=G_{1} \cup G_{2}$, where $G_{1}=D_{n}^{(m-1)}$ and $G_{2}=P_{n-1}$ with 
$V(G-\{v\})=V\left(G_{1}-\left\{v_{1}\right\}\right) \cup V\left(G_{2}-\left\{v_{2}\right\}\right) \cup v_{3}, v_{1}=v_{2}=v_{3}, v_{1} \in V\left(G_{1}\right), v_{2} \in$ $V\left(G_{2}\right)$ and $E(G-\{v\})=E\left(G_{1}\right) \cup E\left(G_{2}\right)$. Let $A$ and $B$ be a $\gamma_{s}$-set of $G_{1}$ and $G_{2}$. Subcase 2.1. If $n=3 p+1, p \geq 1$.

The set $B$ has to contain a vertex $v_{k}$ or $N\left(v_{k}\right), v_{k} \in V\left(D_{n}^{(m)}\right)$, then $\gamma_{s}\left(G_{1}\right)=|A|=(m-1) \gamma_{s}\left(P_{n-1}\right)=m\left\lceil\frac{n-1}{3}\right\rceil$. Thus $\gamma_{s}(G-v)=|A|+|B|=$ $m\left\lceil\frac{n-1}{3}\right\rceil+\left\lceil\frac{n-1}{3}\right\rceil=m\left\lceil\frac{n-1}{3}\right\rceil$. Thus $\gamma_{s}(G-v)=m\left\lceil\frac{n-1}{3}\right\rceil<m\left\lceil\frac{n-1}{3}\right\rceil+1=\gamma_{s}(G)$. Hence $G=D_{n}^{(m)}$ is $\gamma_{s}$-vertex critical for $n=3 p+1, p \geq 1$.

Subcase 2.2. If $n \neq 3 p+1, p \geq 1$.

The set $B$ has to contain a vertex $v_{k}$, then $\gamma_{s}\left(G_{1}\right)=|A|=(m-1) \gamma_{s}\left(D_{n}^{(m-1)}\right)=(m-1)\left\lceil\frac{n-3}{3}\right\rceil+1$. Thus $\gamma_{s}(G-v)=$ $|A|+|B|=(m-1)\left\lceil\frac{n-3}{3}\right\rceil+1+\left\lceil\frac{n-3}{3}\right\rceil=m\left\lceil\frac{n-3}{3}\right\rceil+1$. Thus by Proposition 2.13, $\gamma_{s}(G-v)=\gamma_{s}(G)$. Hence $G=D_{n}^{(m)}$ is not $\gamma_{s}$-vertex critical for $n \neq 3 p+1, p \geq 1$. The result follows from the cases above.

Theorem 2.15. If $G$ is $\gamma_{s}$-vertex critical, then there is no support vertex in $G$ which is adjacent to one or more end-vertices.

Proof. Suppose $v_{1}$ is a support vertex which is adjacent to at least one end-vertex, say $x_{1}$ of a graph $\mathrm{G}$ and let $G_{1}=G-\left\{v_{1}\right\}$. Let $D_{1}$ be a $\gamma_{s}$-set of $G$. Now consider the graph $G_{1}$, since $v_{1}$ is a support vertex in $G$ by Theorem 2.3, $v_{1} \in D_{1}$ and the graph $G_{1}$ is disconnected. Let $A_{1}=\left\{v_{m} / v_{m} \in N\left(v_{1}\right), v_{m} \notin N\left(D_{1}-v_{1}\right)\right\}$.

Case 1. If $\left|A_{1}\right|=1$, then $\gamma_{s}\left(G_{1}\right)=\left|D_{1}\right|-\left|\left\{v_{1}\right\}\right|+\left|A_{1}\right|=\left|D_{1}\right|$. Thus $\gamma_{s}\left(G_{1}\right)=$ $\gamma_{s}(G)$. Which contradicts our assumption.

Case 2. If $\left|A_{1}\right|>1$, then $\gamma_{s}\left(G_{1}\right)=\left|D_{1}\right|-\left|\left\{v_{1}\right\}\right|+\left|A_{1}\right|>\left|D_{1}\right|$. Thus $\gamma_{s}\left(G_{1}\right)>\gamma_{s}(G)$. Which contradicts our assumption.

Hence the proof.

Corollary 2.16. If $G$ is $\gamma_{s}$-vertex critical, then no two support vertices are adjacent.

Proposition 2.17. For any subdivision graph of $K_{n}, \gamma_{s}\left(S\left(K_{n}\right)\right)=n-1, n \geq 3$.

Theorem 2.18. The graph $G=S\left(K_{n}\right)$ is not $\gamma_{s}$-vertex critical for $n \geq 3$.

Proof. Let us assume that $G=S\left(K_{n}\right)$ is $\gamma_{s}$-vertex critical, then for each vertex $v \in V(G), \gamma_{s}(G-v)<\gamma_{s}(G)$. Let us consider the graph $G-\{v\}, v \in V\left(K_{n}\right)$ and $B=\left\{v_{i} / v_{i}\right.$ is a support vertex in $\left.G-\{v\}\right\}$ with $|B|=n-1$ and by Theorem 2.3, $B$ belongs to $\gamma_{s}$-set of $G-\{v\}$ and $\langle G-\{v\}-B\rangle$ is disconnected. Thus $\gamma_{s}(G-v)=|B|=n-1$. By Proposition 2.17, $\gamma_{s}(G-v)=\gamma_{s}(G)$. Which contradicts our assumption. Hence the proof.

Theorem 2.19. The graph $G=S\left(D_{n}^{(m)}\right)$ is $\gamma_{s}$-vertex critical for $n=3 p+2, p \geq 1$ and not $\gamma_{s}$-vertex critical for $n \neq 3 p+2, p \geq 1, m \geq 2, n \geq 4$.

Proof. Case 1. When $n=3 p+2, p \geq 1$.

The graph $S\left(D_{n}^{(m)}\right), n=3 p+2, p \geq 1$ is the graph which is obtained by a subdivision of $D_{n}^{(m)}$ for $n=3 k+2, k \geq 1$. By Theorem 2.14, $G=S\left(D_{n}^{(m)}\right)$ is $\gamma_{s}$-vertex critical for $n=3 p+2, p \geq 1$, 
Case 2. When $n \neq 3 p+2, p \geq 1$.

The graph $S\left(D_{n}^{(m)}\right), n \neq 3 p+2, p \geq 1$ is the graph which is obtained by a subdivision of $D_{n}^{(m)}$ for $n \neq 3 k+2, k \geq 1$. By Theorem 2.14, $G=S\left(D_{n}^{(m)}\right)$ is not $\gamma_{s}$-vertex critical for $n \neq 3 p+2, p \geq 1$.

The result follows from the cases above.

Proposition 2.20. For any subdivision graph of $K_{m, n}$,

$$
\gamma_{s}\left(S\left(K_{m, n}\right)\right)=m+n-1, m \geq n, m, n \geq 2 .
$$

Theorem 2.21. The connected graph $G=S\left(K_{m, n}\right)$ is not $\gamma_{s}$-vertex critical for $m \geq n, m, n \geq 2$.

Proof. Let us assume that the graph $G=S\left(K_{m, n}\right)$ is $\gamma_{s}$-vertex critical, then for each vertex $v \in V(G), \gamma_{s}(G-v)<\gamma_{s}(G)$. Let $V\left(K_{m, n}\right)=V_{1} \cup V_{2}$ where $\left|V_{1}\right|=m,\left|V_{2}\right|=n, A=V\left(S\left(K_{m, n}\right)\right)-V\left(K_{m, n}\right)$. Let us consider the graph $G-\{v\}$, $v \in V_{2}$ and $E=\left\{v_{k} / v_{k}\right.$ is a support vertex in $\left.G-\{v\}\right\}$ with $|E|=m$ and by Theorem 2.3, $E$ belongs to $\gamma_{s}$-set of $G-\{v\}$ and $\langle G-\{v\}-E\rangle$ is disconnected. Thus $\gamma_{s}(G-v)=|E|+\left|V_{2}\right|-|\{v\}|=m+n-1$. By Proposition 2.20, $\gamma_{s}(G-v)=\gamma_{s}(G)$. Which contradicts our assumption. Hence the proof.

Proposition 2.22. For any subdivision graph of $W_{n}$,

$$
\gamma_{s}\left(S\left(W_{n}\right)\right)=\left\lceil\frac{2(n-1)}{3}\right\rceil+1, n \geq 4 .
$$

Theorem 2.23. The graph $G=S\left(W_{n}\right)$ is not $\gamma_{s}$-vertex critical for $n \geq 4$.

Proof. Let us assume that $G=S\left(W_{n}\right)$ is $\gamma_{s}$-vertex critical, then for each vertex $v \in V(G), \gamma_{s}(G-v)<\gamma_{s}(G)$. Let us consider the graph $G-\{v\}, v \in$ $V\left(W_{n}\right), \operatorname{deg}(v)=n-1$ and let $B=\left\{v_{i} / v_{i}\right.$ is an end-vertex $\left.\in G-\{v\}\right\}$ with $|B|=$ $n-1$ and by Theorem 2.3, N(B) belongs to $\gamma_{s}$-set of $G-\{v\}$ and $\langle G-\{v\}-N(B)\rangle$ is disconnected. Thus $\gamma_{s}(G-v)=|B|=n-1$ and $\left\lceil\frac{2(n-1)}{3}\right\rceil+1 \geq n-1$ for $n \geq 4$, by Proposition 2.22, $\gamma_{s}(G-v) \geq \gamma_{s}(G)$. Which contradicts our assumption. Hence the proof.

\section{Split domination EDGe CRitical GRAPhS}

Definition 3.1. A graph $G$ is called edge split critical, if $\gamma_{s}(G+e)<\gamma_{s}(G)$ for every edge e in $\bar{G}$. Thus $G$ is $k-\gamma_{s}$-critical if $\gamma_{s}(G)=k$ for each edge e $\in E(\bar{G})$, $\gamma_{s}(G+e)<k$.

In this entire section, the graph considered should be a connected noncomplete graph.

Lemma 3.2. For any connected graph $G$, the cardinality of the cut-set will remain equal or increases, if an edge $e_{1}$ is added to a graph $G$, where $e_{1} \in E(\bar{G}),(n \geq 4)$.

Proof. Let $V(G)=V(\bar{G})=\left\{v_{11}, v_{12}, \ldots, v_{1 n}\right\}$ and $C$ be a minimum cut-set of a graph $G$. Then the graph $G-C$ is disconnected into at least two components say $G_{1}$ and $G_{2}$ with $V\left(G_{1}\right)=\left\{v_{21}, v_{22}, \ldots, v_{2 n}\right\}$ and $V\left(G_{2}\right)=\left\{v_{31}, v_{32}, \ldots, v_{3 n}\right\}$. Let us consider the graph $G_{1}=G+e_{1}$, where $e_{1}=\left(v_{2 i}, v_{2 j}\right) \in E(\bar{G})$ for $i \neq j$. Then 
the graph $G_{1}$ is disconnected and hence the cut-set remains same. Otherwise if $e_{1}=\left(v_{2 i}, v_{3 j}\right) \in E(\bar{G})$, then the graph $G_{1}$ is connected and thus we need a vertex of $G$ to make the graph $G_{1}$ disconnected. Hence the cardinality of the cut-set will remains same or increase, if an edge $e_{1}$ is added to a graph $G$, where $e_{1} \in E(\bar{G})$. This completes the proof.

Lemma 3.3. Every minimum split domination set must contains the cut-set of a graph $G$.

Theorem 3.4. There is no edge critical graph with respect to split domination.

Proof. Let $D_{1}$ be a $\gamma_{s}$-set of $G$. Let us consider the graph $G+e$, where $e \in E(\bar{G})$. By Lemma 3.1 and Lemma 3.2, $\gamma_{s}(G+e) \geq\left|D_{1}+1\right|$. Hence with respect split domination, $G$ is not edge critical.

Theorem 3.5. For any connected graph $G$,

$$
\gamma_{s}(G) \leq \gamma_{s}(G+e) \leq \gamma_{s}(G)+1, e \in E(\bar{G}),(n \geq 4) .
$$

Proof. Let $D$ and $D_{1}$ be a $\gamma_{s}$-set of $G$ and $G+e, e \in E(\bar{G})$. From Lemma 3.1 and $3.2, \gamma_{s}(G) \leq \gamma_{s}(G+e)$. Let $A$ be a minimum cut-set of a graph $G$. By Lemma $3.2, A \subseteq D$. The graph $G-A$ will be disconnected into $k$ number of components $G_{1}, G_{2}, G_{3}, \ldots, G_{k}$.

Case 1. If $e=v_{1} v_{2} \in E(\bar{G}),\left\{v_{1}, v_{2}\right\} \in V\left(G_{1}\right)$, then $\gamma_{s}(G)=\gamma_{s}(G+e)$.

Case 2. If $e=v_{1} v_{2} \in E(\bar{G}), v_{1} \in V\left(G_{1}\right), v_{2} \in V\left(G_{2}\right)$, then at least one of $\left\{v_{1}\right\}$ or $\left\{v_{2}\right\}$ must belong to $D_{1}$ to make the graph $G+e$ disconnected. Thus $\gamma_{s}(G+e)=$ $\left|D_{1}\right|=|D|+\mid\left\{v_{1}\right\}$ or $\left\{v_{2}\right\} \mid=\gamma_{s}(G)+1$. Hence $\gamma_{s}(G+e) \leq \gamma_{s}(G)+1$.

This completes the proof.

\section{Conclusion}

In this paper, it is verified that some standard graphs along with any connected graphs are split domination vertex critical or not. In the military network database, they will have an idea what type of network they can have in such a way that the network or part of the network is critical. In the network if any of the domination members is not available, they can reduce the domination members by at least one.

\section{REFERENCES}

[1] Arumugam, S. and Paulraj J. J., Domination in Subdivision graphs, Journal of Indian Mathematical Society 62(1-4) (1996), 274-282.

[2] Brigham, R. C., Chinn P. and Dutton R. D., Vertex domination critical graphs, 18 (1988), 173-179.

[3] Chen, X. G., Sun, L. and Ma, D., Connected Domination Critical graphs, Applied Mathematics. 17 (2004), 503-507.

[4] Harary, F., Graph theory, Addison-wesley, reading Mass 1969.

[5] Haynes, T. W., Hedetniemi, S. T. and Slater, P. J., Fundamental of domination of graphs, Marcel Dekker Inc., 1988. 
[6] Kulli, V. R. and Janikiram B., The split domination number of a graph, Graph Theory Notes of New York Academy of Sciences, 32 (1997), 16-19.

[7] Lemanska, M. and Patyk, A., Weakly Connected Domination Critical graphs, Opuscula Mathematica, 28 No. 3 (2008), 325-330.

[8] Sumner, D. P., Critical concepts in domination, Discrete Math., 86 (1990), 33-46.

[9] Summer, D. P. and Blitch P., Domination critical graph, Journal of combinatorial theory series $B, 34$ (1983), 65-76.

[10] Tamizh C. T. and Robinson C. S., A note on split domination number of a graph, Journal of Discrete Mathematical Sciences and Cryptography, 12:2 (2009), 179-186. 